\title{
INCIDENCIA DEL RATING EN LA CALIDAD DE LOS CONTENIDOS PERIODÍSTICOS COMUNITARIOS DE DOS CANALES TELEVISIVOS, 2014
}

\author{
INFLUENCE OF RATING ON COMMUNITY JOURNALISTIC CONTENT QUALITY \\ OF TWO TV CHANELS, 2014
}

\section{MARITZA FILOMENA CARVAJAL LITUMA}

Facultad de Filosofía, Letras y Ciencias de la Educación. Universidad Católica de Santiago de Guayaquil maritza.carvaja@cu.ucsg.edu.ec

RESUMEN

La investigación tuvo como objetivo establecer cómo incide el rating en la calidad de los contenidos periodísticos comunitarios en los noticieros de Canal Uno y RTS, a través de un estudio descriptivo y correlacional para la creación de un Observatorio Ético Virtual de la cobertura comunitaria, con cuenta en Twitter. La metodología incluyó el análisis de contenido, a partir de una selección de principios del Periodismo y valores éticos, el grupo focal con expertos en periodismo de la comunidad, la entrevista con las directoras de los noticieros y la firma que mide la sintonía, lbope; la encuesta para el establecimiento de la percepción de los televidentes y la observación no participante para la descripción del formato de los noticieros. En los noticieros de la comunidad de los dos canales de televisión estudiados, Canal Uno y RTS, se da gran importancia al rating y a partir de esa consideración se privilegia la forma, tanto para el formato del programa noticioso, como para el abordaje de las noticias en detrimento de la calidad de las informaciones. Al término se creó el Observatorio Ético Virtual sobre noticias de la comunidad, MiradaUCSG, con cuenta en Twitter.

PALABRAS CLAVE: periodismo, noticias comunitarias, audiencias, principios periodísticos, ética de la profesión.
ABSTRACT

The research was aimed to determine the influence of the rating on the quality of community journalistic content of Canal Uno and RTS news through a descriptive and correlational study so as to create a Virtual Ethical Observatory of the community by means of a Twitter account. Content analysis was considered in the methodology through the selection of the basic principles of journalism; focus groups, interviews, and surveys were used as well for the collection of information and for the non-participant observation. The results retrieved allowed the researcher to find out that, regarding community news, the two TV channels sampled, Canal Uno and RTS, place great importance on the rating issue privileging the form of both the format of the news program and the approach to public broadcasting, thus becoming detrimental to the quality of the information. The creation of a Virtual Ethical Observatory, Mirada UCSG, has emerged as a result of this research. This observatory is devoted to community news, has a Twitter account, and has been active for three months now.

KEYWORDS: journalism, community news, audiences, journalistic principles, professional ethics. 
INTRODUCCIÓN

Desde la década de los 80 del siglo XX, cuando comenzaron las primeras experiencias de periodismo comunitario, en Estados Unidos y América Latina, el segmento ha ido ganando espacio de manera paulatina en los noticieros de televisión. En aquella época, los canales emitían un par de informaciones sobre los temas vinculados con la comunidad, como la calidad de los servicios básicos, las condiciones de los parques y ambientes, entre otros, y el resto de noticias estaban relacionadas con la política, la economía, judiciales, deportes y cultura, entre otros. Ahora el segmento tiene un espacio propio, de similar interés que las noticias políticas, económicas y judiciales.

En Ecuador, la primera experiencia del segmento comunitario se presentó en años previos, en Telecentro Canal 10, en el noticiero El Observador. Durante los años 60 y 70 ese noticiero asignó un espacio al segmento comunitario La Calle lo Contó, que dio fama a su presentador, Antonio Hanna Musse.

Espinoza (2013) recuerda que en Ecuador, entre las décadas de los ochenta y noventa, los noticieros comunitarios de la televisión estaban adormilados y no cumplían con los objetivos principales de aporte a la comunidad, mientras que los noticieros nacionales e internacionales de corte tradicional primaban en la parrilla televisiva.

Luego, en 1993, surgió el espacio Ventana a la Calle, conducido por el periodista Kenneth Carrera en Guayaquil, que se transmitía en el desaparecido Canal 4, ahora RTS. Carrera, con un escritorio ubicado en una esquina popular de la ciudad, recibía a la gente que denunciaba sus problemas comunitarios en televisión.

A partir del año 2000, los noticieros comunitarios resurgen y se posicionan dentro de los noticieros de televisión (Espinoza, 2013).

Por la buena acogida, en la actualidad los canales cuentan con espacios de una duración de media a una hora, en diferentes tipos de formatos, que incluyen entrevistas en el estudio, enlaces externos para entrevistas en el lugar de los hechos, elaboración de noticias de temas comunitarios, el reporte del Sistema Ojos de Águila, entre otras secciones. En los noticieros el periodista realiza coberturas en los barrios o comunidades para palpar de cerca los problemas del sector e insertar las voces de los ciudadanos comunes en las narraciones periodísticas.

Algunas experiencias de noticieros comunitarios en Ecuador son, Contacto al Amanecer,
Contacto en la Comunidad y Ayúdame Henry, en Ecuavisa; Noticiero 24 Horas en la Comunidad, de la cadena televisiva Teleamazonas, el Noticiero Bien Informado de TC Televisión, Ecuador TV Noticias en la Comunidad y los dos noticieros, objeto de este estudio, Noticiero de la Comunidad de Canal Uno y La Noticia en la Comunidad, de la cadena RTS. Algunos han desaparecido y otros se han transformado.

En el ámbito comunitario también han existido experiencias en la plataforma radial. Un ejemplo fue el programa radial del "Maestro Juanito", quien realizaba entrevistas en la calle, de manera similar a Antonio Hanna y Kenneth Carrera. Luego apareció el programa radial de Gustavo Herdoiza, que lo catapultó a la Alcaldía de Quito (Espinoza, 2013).

Los diarios impresos también tienen sus secciones comunitarias, en las que abordan noticias vinculadas con la ciudad, desde la perspectiva del ciudadano común. Y en las versiones en la plataforma en la Internet, se apoyan en las redes sociales para acercar el abordaje noticioso a la realidad cotidiana de los habitantes.

Empero, en la realidad ecuatoriana contemporánea, en algunos casos, el tratamiento periodístico de los noticieros de la comunidad se orienta más al entretenimiento que a la información.

Tal problemática motiva esta investigación porque las noticias son fundamentales para un público que busca conocer información relevante en el entorno local, nacional o internacional. En los noticieros, los integrantes de la sociedad también tienen un espacio que los acerca a las autoridades, y los periodistas se convierten en el puente entre ambos sectores, de allí que el tratamiento de los hechos debe considerar cuatro puntales: la ética de la profesión, los principios y reglas del Periodismo y el respeto a los públicos y audiencias.

\section{MATERIALES Y MÉTODOS}

La investigación estuvo en el campo no experimental, y aplicó un enfoque mixto. La investigación es de tipo descriptivo-correlacional.

El universo de estudio incluyó a las noticias de los telediarios de la comunidad de los canales RTS, 1.382 en total y de Canal Uno, 1507 en total, en las emisiones de lunes a viernes, durante tres meses a saber, marzo, abril y mayo de 2014. De esa población la muestra abarcó 474 noticias de temas catalogados como comunitarios, conforme la acepción asimilada para este estudio. Y la muestra fue de tipo no probabilístico porque 
se escogieron únicamente las que aplicaban el criterio comunitario.

Como técnicas de acopio de información, la investigación utilizó:

- Revisión bibliográfica para la selección de los criterios a utilizar en este estudio, a partir de los principios, postulados éticos y reglas del Periodismo.

- Análisis de contenido para el estudio de las noticias de la comunidad de los dos noticieros, durante los meses de marzo, abril y mayo de 2014.

- Entrevista para obtener las versiones oficiales de las directoras de los dos noticieros de TV de Canal Uno y RTS, Carola Artieda y Mariuxi Padilla, respectivamente.

- Grupo focal para los cuatro expertos en Periodismo de la comunidad, Ronald Soria, Marlon Puertas, Alcides Montilla y Silvia Coello.

- Encuesta para establecer tendencias de opinión en los habitantes de Guayaquil, respecto de los noticieros de la comunidad en general y de los de Canal Uno y RTS en particular.

- Observación no participante para la descripción del formato de presentación de los dos noticieros, además de la comparación del tiempo de emisión de los noticieros, la contabilización de las publicidades.

Para la sistematización de la información se utilizaron bases de datos, en Excel, con el propósito de registrar de manera diaria el análisis del contenido de las muestras de las noticias comunitarias de Canal Uno y de RTS.

Una tercera base de datos, en Excel, se utilizó para la tabulación de los resultados de las 22 preguntas incluidas en el cuestionario aplicado a 778 habitantes de 20 sectores del norte y sur de Guayaquil.

Para la presentación de los resultados se utilizaron tablas comparativas, gráficas en Excel y matrices comparativas.

\section{RESULTADOS}

La evidencia obtenida en esta investigación confirma la hipótesis respecto a que el rating incide en negativo en la calidad de los contenidos periodísticos de los noticieros de la comunidad, porque a partir de él, las directoras de los noticieros favorecen la forma en la presentación de los informativos en los casos estudiados, a saber, Noticiero de la Comunidad de Canal Uno y La Noticia en la Comunidad de RTS. Y también en el abordaje de las noticias, al incorporar en variadas ocasiones el componente sensacionalista, por medio de la musicalización, la repetición de escenas, un abordaje extendido del hecho noticioso, sin que amerite desde la consideración de los valores-noticia para impresionar al televidente. Dichos puntos fueron descubiertos de manera adicional con el análisis de contenido y la observación no participante, para cada noticiero.

En los noticieros se sacrifica el tiempo otorgado al contenido para concedérselo a la interacción jocosa entre los presentadores, y los comentarios-opiniones de éstos, antes o después de difundida la noticia. En el caso de RTS, para la interacción entre los presentadores y animaciones que aparecen sobrepuestas en la pantalla.

La investigación demuestra que la competencia, inherente al periodismo, entre los dos medios de comunicación, se da respecto a qué canal ofrece el noticiero más entretenido, por la diversión o el espectáculo que se desarrolla en el estudio y no a partir de qué canal ofrece la mejor información posible, como consecuencia de una investigación en las fuentes de información pertinentes y una narración coherente y sustentada. Ello pese a que $51,8 \%$ de un total de 778 encuestados en los 20 sectores del norte y sur de Guayaquil, que pertenecen a las circunscripciones 1 y 2, dijeron estar más interesados en el contenido de los noticieros de la comunidad, en la información en sí misma, que en el formato informal, los presentadores de los noticieros, o las bromas y chistes que allí surgen, como se evidencia en la figura 1.

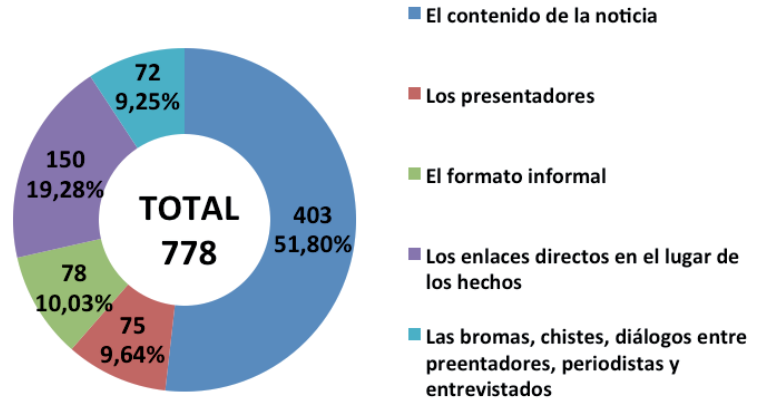

Figura 1. Resultados de los factores que más atraen de los noticieros de la comunidad, circunscripciones 1 y 2 .

Por su parte los expertos en periodismo, quienes participaron en los grupos de enfoque, a saber, Silvia Coello, Alcides Montilla, Marlon Puertas 
y Ronald Soria, expresaron criterios disímiles. Unos consideraron que el formato informal (la interacción jocosa y entretenida entre los presentadores del noticiero) pudiera aceptarse, siempre y cuando el tratamiento que se ofrezca en las noticias, a saber el contenido periodístico, aplique los principios y la ética de la profesión. Mientras que a otros expertos, el formato, que incluye bailes y bromas entre presentadores, les desagradó por completo, con el argumento que los noticieros no deben mezclarse o confundirse con formatos de talk shows, porque tienen otra naturaleza.

En las dos emisiones de los noticieros de la comunidad de RTS, las noticias de la comunidad representaron $34,29 \%$, del total de las noticias difundidas en los tres meses de estudio (marzo, abril y mayo), acorde con los criterios seleccionados para este estudio, como se muestra en la tabla 1.

En las tres emisiones de los noticieros comunitarios de Canal Uno, en los tres meses de estudio (marzo, abril y mayo), las noticias de la comunidad representaron $40,41 \%$, como también se refleja en la tabla 1.

\begin{tabular}{|c|c|c|c|c|}
\hline \multirow[t]{2}{*}{ MESES } & \multicolumn{2}{|c|}{ CANAL UNO } & \multicolumn{2}{|c|}{ RTS } \\
\hline & $\begin{array}{l}\text { Total de } \\
\text { Noticias }\end{array}$ & $\begin{array}{r}\text { Noticias } \\
\text { Comunitarias }\end{array}$ & $\begin{array}{r}\text { Total } \\
\text { noticias }\end{array}$ & $\begin{array}{r}\text { Noticias } \\
\text { comunitarias }\end{array}$ \\
\hline $\begin{array}{l}\text { Marzo de } \\
2014\end{array}$ & 539 & 195 & 417 & 125 \\
\hline $\begin{array}{l}\text { Abril de } \\
2014\end{array}$ & 478 & 225 & 480 & 160 \\
\hline $\begin{array}{l}\text { Mayo de } \\
2014\end{array}$ & 490 & 189 & 485 & 189 \\
\hline $\begin{array}{l}\text { Total } \\
3 \text { meses }\end{array}$ & 1.507 & $\begin{array}{r}609 \\
(40,41 \%)\end{array}$ & 1.382 & $\begin{array}{r}474 \\
(34,29 \%)\end{array}$ \\
\hline
\end{tabular}

Al comparar los dos canales, RTS y Canal Uno, se estableció que el criterio con mayor cantidad de incumplimientos en ambos canales fue el equilibrio. Así, en RTS el incumplimiento representó $43 \%$ del total 474 noticias de la muestra; mientras que en Canal Uno equivalió a $32 \%$, de un total de 609 noticias de la muestra, como consta en la Tabla 2. El criterio de equilibrio obliga a los periodistas al contraste de las fuentes vinculadas con el hecho noticioso (Benavides \& Quintero, 2004).

En cuanto al criterio de atribución, en Canal Uno, el porcentaje de incumplimientos fue de $32 \%$, del total de 609 noticias; mientras que en RTS llegó a 5\%, del total de 474 noticias, como aparece en la Tabla 3. Ello significa que ese por- centaje de notas no identificaba a las fuentes de información.

El tercer criterio con más incumplimientos es el lenguaje. En RTS, el porcentaje de incumplimientos ascendió a $35 \%$ del total de noticias, mientras que en Canal Uno fue de 4,43\%. En periodismo, el lenguaje a utilizar es el formal, no el coloquial, ni el uso de tecnicismos sin explicar.

La comparación de los demás criterios, en valores relativos, puede observarse a continuación en la Tabla 2, y luego en valores absolutos, por canal, en las Figuras 2 y 3.

TABLA 2. COMPARATIVA DE LOS INCUMPLIMIENTOS DE LOS CRITERIOS PERIODÍSTICOS DE ANÁLISIS EN CANAL UNO Y RTS

\begin{tabular}{lrr}
\hline CRITERIOS & CANAL UNO & RTS \\
\hline Equilibrio & $32 \%$ & $43 \%$ \\
\hline Exactitud & $20,68 \%$ & $1 \%$ \\
\hline Atribución & $32 \%$ & $5 \%$ \\
\hline Lenguaje & $4,43 \%$ & $35 \%$ \\
\hline Contexto & $20,68 \%$ & $5 \%$ \\
\hline Calidad de las preguntas & $13,95 \%$ & $14 \%$ \\
\hline Ética & $0,49 \%$ & $11 \%$ \\
\hline Dramatismo* & $12,15 \%$ & $23 \%$ \\
\hline Total de noticias consideradas & 609 & 474 \\
\hline *A efectos de la revisión del criterio de dramatismose considera la incorporación \\
de componentes que lo acentúan, a saber, se efectúa una revisión diferente a los \\
otros criterios. & \\
\hline
\end{tabular}

Una diferencia en las opiniones entre los expertos en periodismo y las directoras de los noticieros radica en la incorporación de música de fondo en las noticias. Alcides Montilla, Ronald Soria, Marlon Puertas y Silvia Coello coinciden en que la musicalización de las noticias afecta a la credibilidad, y en ocasiones cuestionan la ética, porque acentúa el drama para capturar la empatía del televidente, mientras que la directora de los noticieros de RTS indica que no afecta a la ética, ni revictimiza a los afectados. La directora del noticiero de Canal Uno afirmó que es un recurso para mantener la expectativa del televidente.

El análisis de contenido y la observación no participante permitió establecer que la musicalización se utiliza tanto para acentuar el drama, como para mantener la expectativa del televidente.

El 34\% de los 778 encuestados en los 20 sectores del norte y sur de Guayaquil considera que el tratamiento de las informaciones del noticiero de RTS, a veces incorpora el dramatismo. El 


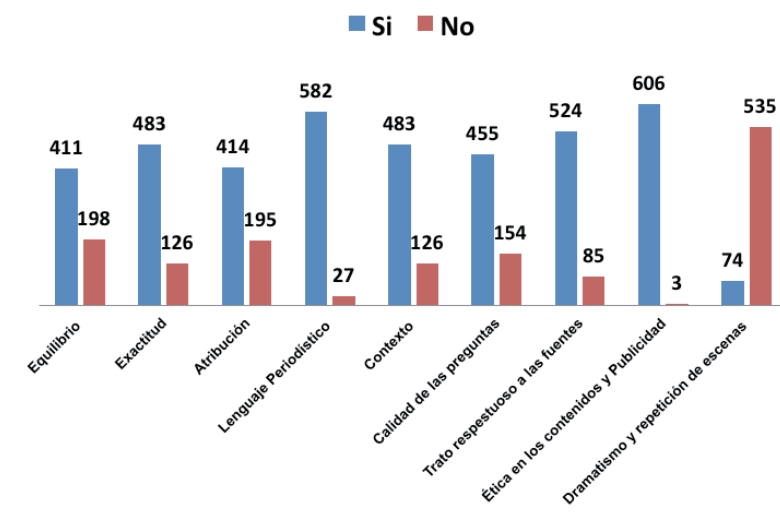

Figura 2. Consolidado de tres meses de análisis de contenido de Canal Uno.

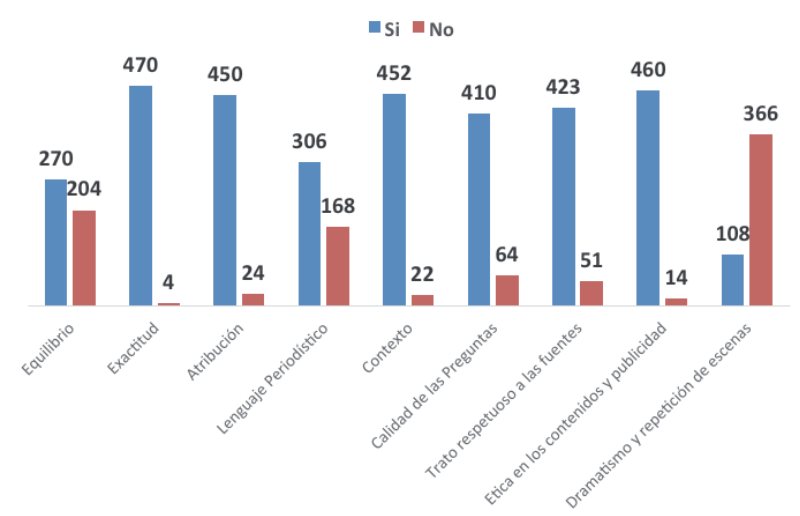

Figura 3. Consolidado de tres meses de análisis de contenido de RTS.

análisis de contenido aplicado a RTS durante los meses de marzo, abril y mayo, obtuvo como resultado que la inserción del dramatismo se amplió del 20\% de las noticias en marzo, al 26\% en mayo.

El 37\% de los 778 encuestados en el norte y sur de Guayaquil afirmó que el abordaje de las informaciones del noticiero de la comunidad de Canal Uno sí tiene dramatismo. El análisis de contenido aplicado a las informaciones de los noticieros de la comunidad de Canal Uno se logró establecer que la inserción del dramatismo en el tratamiento de las informaciones se amplió del 5\% de las noticias registradas en marzo de 2014 al 18\% de las noticias registradas en mayo.

En ese contexto, los expertos en Periodismo expresaron en varias oportunidades que el $\mathrm{Pe}$ riodismo es una profesión y como tal tiene unos principios, que no pueden compararse con otro tipo de programas de corte dramático, ni con el cine.

Los cuatro expertos en Periodismo, Alcides Montilla, Ronald Soria, Marlon Puertas y Silvia Coello, consideran que los noticieros de Canal Uno y RTS, tienen el membrete de comunitarios, pero su contenido, a saber buena parte de los temas seleccionados, y su abordaje están lejos de ser comunitarios.
Para ellos, las notas de la comunidad deben partir del ciudadano, de los barrios, de la cotidianidad, con notas positivas, además de las negativas, que reflejen de alguna manera el empoderamiento del habitante para contribuir con la solución de problemas en su entorno.

Los noticieros de la comunidad de los canales RTS y Uno, objeto de estudio de esta investigación, tienen la preferencia de los encuestados en los 20 sectores del norte y sur de Guayaquil, respecto del resto de los noticieros de la comunidad de los canales ecuatorianos; y se ubican en el primer lugar con el $28 \%$ de las preferencias, y en segundo lugar con $27 \%$, respectivamente

El $40 \%$ de los 778 encuestados en los 20 sectores del norte y sur de Guayaquil identificó a los temas vinculados con servicios básicos (agua potable, alcantarillado, electricidad, entre otros) como los que más se difunden en los noticieros de la comunidad de Ecuador. Esa misma temática fue luego seleccionada como la de mayor importancia para los moradores en sus respectivas zonas.

Como corolario de esta investigación nació el Observatorio Ético Virtual MiradaUCSG, que a octubre de 2015 alcanzó más de 4.000 visitas. Al $41 \%$ de los 778 encuestados en los 20 sectores de las zonas sur y norte de Guayaquil les interesó la posibilidad de participar en un observatorio de medios de comunicación.

DISCUSIÓN

La investigación realizada a partir de los noticieros de la comunidad de los canales Uno y RTS revela que el supuesto que las personas de menores ingresos están interesadas en el formato informal de los noticieros de TV es errada. Este punto nos permite reflexionar a su vez sobre la justificación que acostumbran esgrimir los productores y directores de noticieros y otros programas de televisión, no solo en Ecuador sino también en el mundo: que la televisión es el reflejo de lo que exige la sociedad. Creemos que ese pretexto ha sido extrapolado al caso específico de los noticieros de televisión, cuyos productores y directores tienen presente a las mediciones del rating como una guía u orientador en la toma decisiones, tanto para los contenidos periodísticos como para los formatos de presentación de noticieros y noticias.

Consideramos que el argumento de las mediciones del rating, utilizado como excusa por los directivos de los canales para privilegiar la forma, tanto en la producción de los noticieros como en el abordaje de las informaciones, ha logrado deteriorar los contenidos periodísticos 
y desprestigiar a una profesión que tiene como compromiso con la sociedad, aportar desde la investigación de temas de trascendencia, a la resolución de problemas socioeconómicos, políticos y de otra índole, con una puesta en escena que asimile la verificación y el cotejo de los datos, el equilibrio y la contextualización, entre los más importantes.

En la actualidad, en Ecuador la competencia entre los medios de comunicación se da desde la perspectiva de quién ofrece el mejor y más novedoso formato de noticiero, quien ofrece el mejor espectáculo televisivo y no quién ofrece la mejor construcción noticiosa, a partir de los principios y la ética de la profesión. De allí que se insertan bromas subidas de tono, bailes, canto, animaciones, y otra suerte de artilugios que buscan el entretenimiento y no la calidad informativa. Creemos que, si la situación persiste, el deterioro de los informativos de televisión, de corte comunitario, continuará en detrimento de los televidentes, en particular, y de la sociedad en general, porque en lugar de orientar, y contribuir a la interpretación y lectura crítica de los hechos, situaciones o fenómenos noticiosos generará desinformación y tornará inútiles a los productos periodísticos.

Lo que hasta ahora ofrecen los noticieros de la comunidad acrecienta la deuda con la sociedad, porque además de la mínima cantidad de noticias de corte comunitario que difunden, ellas tienen un abordaje que favorece el sensacionalismo que hace tabla rasa de los principios y postulados éticos del Periodismo, así como de la Teoría de la Responsabilidad Social de los Medios, que orientó este estudio.

Pensamos que, el abordaje sensacionalista de las informaciones es el camino fácil que han escogido algunos noticieros de TV guiados por el rating para la obtención de mayores ingresos, desde el avisaje publicitario.

Si bien, los medios de comunicación necesitan ingresos, y están en su derecho de conseguirlos porque necesitan financiar sus costos de operación que son cuantiosos, creemos que ese requerimiento urgente no debe convertir en una alfombra a una profesión tan noble como el Periodismo, porque a la larga ello redundará en su propio perjuicio, como puede establecerse de la percepción que tienen los consumidores sobre la credibilidad de los medios de comunicación en diferentes partes del mundo, registrada en encuestas efectuadas por diferentes organizaciones y en diferentes espacios-tiempo.

\section{CONCLUSIONES}

En las dos emisiones de los noticieros de la $\mathrm{Co}^{-}$ munidad de RTS, las noticias de la comunidad en los tres meses de estudio, esto es marzo, abril y mayo sumaron 474 , que representaron $34,29 \%$ del total de noticias difundidas en los tres meses, a saber 1.507 noticias.

En las tres emisiones de los noticieros de la comunidad de Canal Uno, las noticias de la comunidad en los tres meses de estudio, esto es marzo, abril y mayo sumaron 609, que representaron $40,41 \%$ del total de las difundidas en los tres meses, a saber 1.382 noticias.

Los criterios periodísticos seleccionados para el estudio fueron: equilibrio, exactitud, atribución, lenguaje periodístico, contexto, la calidad de las preguntas, el trato para las fuentes de información, la ética, contenidos y publicidad y el dramatismo y repetición de escenas.

El mayor rating del noticiero de la comunidad de RTS fue 10,9 puntos en la emisión de la noche, el 11 de marzo de 2014.

El mayor rating de Canal UNO fue de 3,2 puntos en el noticiero de la mañana, en los días 8 y 21 de abril de 2014 .

El noticiero de la mañana de RTS recibe más publicidades, que el de la noche, a saber entre 30 y 55 publicidades diarias, dependiendo del mes. Mientras en Canal Uno, el avisaje varió entre 4 y 15 publicidades diarias, en el noticiero de la mañana, en el lapso estudiado, esto es entre marzo y mayo de 2014.

En ambos canales el rating sí es considerado, según las directoras de los noticieros de la comunidad de los canales Uno, Carola Artieda, y de RTS, Mariuxi Padilla; y el estudio logró establecer que tal consideración sí afecta la calidad de las noticias, porque se privilegia el formato, al ofrecerle tiempo a la interacción entre los conductores de los respectivos noticieros, o de éstos con figuras animadas. En unos casos con un lenguaje coloquial, grosero, bromas, comentarios personales, cantos o movimientos de baile, o simulación de peleas, o amenazas a fuentes externas.

El criterio con mayor cantidad de incumplimientos en Canal Uno y RTS, es el equilibrio. En RTS el incumplimiento representó 43\% del total 474 noticias; y en Canal Uno equivalió a $32 \%$, de un total de 609 noticias.

Otro criterio con más incumplimientos fue el lenguaje. En RTS, el porcentaje de incumplimiento ascendió a 35\% del total de noticias, mientras que en Canal Uno fue de $4,43 \%$. 
En cuanto al criterio de atribución, en Canal Uno, el porcentaje de incumplimiento fue de $32 \%$, del total de 609 noticias; mientras que en RTS llegó a 5\%, del total de 474 noticias.

En cuanto al maridaje entre publicidades y contenidos noticiosos, a saber el criterio de la ética, no se registraron faltas en ninguno de los dos canales.

Empero, el criterio de la ética en los contenidos estuvo también ligado al sesgo en favor de alguna fuente de información o la exageración en el abordaje de algún hecho noticioso, y allí hubo tres casos en Canal Uno, en los tres meses estudiados y 14 casos en RTS.

Un criterio que cabe rescatar, porque está relacionado con el abordaje de las noticias es el drama, que reivindica la importancia que se otorga a la forma y no al fondo en los contenidos, y atenta a la ética.

En este punto $12,15 \%$ (74 noticias) del total de 609 noticias difundidas en los tres meses de estudio (marzo, abril y mayo) en Canal Uno, registró alguno de los componentes que acentuó el drama.

En cambio, en RTS, 22,78\% (108 noticias) del total de 474 noticias difundidas en los tres meses de estudio (marzo, abril y mayo) registró alguno de los componentes que acentuó el drama.

Con relación al Cuarto Objetivo, se logró establecer que mientras las directoras de los dos canales estudiados, Canal Uno y RTS, defienden la importancia del formato para las emisiones de las mañanas, los cuatro expertos en Periodismo Comunitario indicaron que los noticieros de televisión no son talkshows para propiciar espectáculos informativos, porque tienen una responsabilidad para con los públicos y audiencias.

La encuesta aplica a 778 habitantes de 20 sectores en el norte y sur de Guayaquil, provenientes de las circunscripciones 1 y 2 indicaron que sí les gustaría participar en el Observatorio Ético Virtual.

El Observatorio Ético Virtual de la Universidad Católica Santiago de Guayaquil, MiradaUCSG nació en noviembre de 2014, y su sitio en la Internet es https://miradaucsg.wordpress. com/, que hasta octubre de 2015 había recibido más de 4.000 visitas.

AGRADECIMIENTOS

Agradezco a las y los estudiantes que colaboraron en este trabajo de investigación, a los cuatro expertos en Periodismo y a las directoras de los noticieros de televisión por su tiempo para explicar la producción de los noticieros.

\section{REFERENCIAS BIBLIOGRÁFICAS}

Asamblea Nacional (2013). Ley Orgánica de Comunicación. Registro Oficial de Ecuador. Recuperado de: http://www.supercom.gob.ec/ sites/default/files/document/ley_organicacomunicacion.pdf

Abad, G. (2013). Medios, periodismo y responsabilidad social: en busca de políticas públicas en el Ecuador. Revista Chasqui No. 122. Recuperado de: http://repositorio. ciespal.org:8080/bitstream/123456789/1316/1/ CIESPALChasqui_Medios,_periodismo_y_ responsabilidad_social_en_busca_de_ pol\%C3\%ADticas_p\%C3\%BAblicas_en_el_ Ecuador.pdf

Antezana, L. (2010). El Noticiero Televisivo y su Rol Social: El Caso Chileno. Revista Razón y Palabra. №71.VOL.15. Recuperado de: https:// razonypalabra.org. mx/N/N71/VARIA/4\%20 ANTEZANA_REVISADO.pdf

Arroyave, J; Blanco, I. (2005). Cómo perciben los periodistas su profesión: Entre el agotamiento y la fascinación. Recuperado de: http://rcientificas.uninorte.edu.co/index. php/investigacion/article/viewFile/1004/630

Baesso, M; Ferraze, C (2013). The right to information and the (still limited) citizen's space in Journalism popular printed. Revista Intercom-RBCC. Volumen 36. N. 1. Recuperado de: http://www.redalyc.org/ pdf/698/69831050003.pdf

Baran, S. (2005). Comunicación masiva en Hispanoamérica. Cultura y literatura mediáticas. 3ra edición. Mc Graw Hill.

Benavides, J; Quintero, C. (2004). Escribir en prensa. 2da edición. Pearson Comunicación: Madrid.

Cáceres, M. (2007). Telerrealidad y aprendizaje social. Revista Ícono. Volumen 14 N. 9. Recuperado de: http://www.icono14.net/ojs/index. php/icono14/article/view/375/251

Carvajal, M. (2014). La relación entre el desarrollo de competencias en el proceso de enseñanza aprendizaje del Periodismo y el desempeño en las prácticas pre profesionales en los medios de comunicación. Revista Alternativas. Vol 15, N.2. ISSN 13901915.

CIS. (2013). Latinobarómetro 2013. España: CIS. Recuperado: http://www.cis.es/cis/ export/sites/default/-Archivos/Marginales/3000_3019/3007/es3007mar.pdf

Cordicom (2014). Reglamento para la medición de sintonía de los canales de televisión. Consejo de Regulación y Desarrollo de la Información 
y Comunicación. Recuperado 18/07/2014 de www.cordicom.gob.ec

Crespo, (2013). La ética del cuarto poder. La nueva realidad del Periodismo. Recuperado de: http://www.tdx.cat/bitstream/handle/10803/123277/TMCA.pdf?sequence $=1$

Dominick, J. (2006). La dinámica de la comunicación masiva. Los medios en la era digital. 8va. Edición. Mc Graw Hill.

El Comercio (2014). Chiriboga dice que sí es su voz la de la grabación filtrada Recuperado de: http://www.elcomercio.com.ec/deportes/ luischiriboga-audio-fef-ligaprofesionalfutbolriobamba.html.

Fundación para el Nuevo Periodismo Iberoamericano. (2008). La otra cara de la libertad. La responsabilidad social empresarial en los medios de comunicación de América Latina. Recuperado de: http://www.fnpi.org/ uploads/media/P_FNPI_RSE_La_otra_cara_ de_la_libertad.pdf

González, M. (2010). Ética Periodística y el autocontrol: convergencias de cinco países andinos. Revista Chasqui N. 111. Recuperado: http://chasqui.ciespal.org/index.php/ chasqui/article/viewFile/326/326

Gómez, J; Hernández, J; Gutiérrez, L; Arango, G; Franco, A. (2010). Los noticieros de la televisión colombiana "en observación". Una mirada desde la academia a la estructura, cobertura y contenidos de los tele informativos de la televisión abierta en Colombia. Revista Palabra Clave. Volumen 13 N. 2. Recuperado: file://D:/user\%20files/Downloads/1762-7793-1PB.pdf

Grijelmo, A (2008). El Estilo del Periodista. 16va edición. Taurus: Madrid.

Ibope (2013). Metodología para medir el rating. Recuperado. http://www.ibope.com.ec/ ibopetime/

Myers M. (2011). Voces desde las villas. Radios comunitarias en el mundo en vías de desarrollo. Recuperado de: http://cima.ned. org/sites/default/files/CIMA-Community_ Radio-Report_Final\%20-\%2006-30-11.pdf

Montoya, C. (2009). Medios de comunicación y organizaciones de la sociedad civil: fortaleciendo alianzas para la democracia y los derechos humanos en América Latina. Revista Signoy Pensamiento. Volumen 28. N. 55. http:// www.redalyc.org/pdf/860/86020246012.pdf
Pomares, R. (2010), Las noticias de la Comunidad Valenciana en televisión Estudios sobre el mensaje periodístico. Recuperado de: http:// revistas.ucm.es/index.php/ESMP/article/ viewFile/ESMP1010110395A/11487

Proyecto para un periodismo de excelencia. (2003) ¿Importa la Propiedad Local de las noticias de Televisión? Recuperado de: http://www. journalism.org/2003/04/29/does-ownershipmatter-in-local-television-news/

Quintana, R. (2005). La responsabilidad de los programadores de TV. Revista Comunicar. Volumen 25. Recuperado de: http://www. redalyc.org/pdf/158/15825034.pdf

Redondo, M. (2011). El sensacionalismo y su penetración en el sistema mediático español.

Sáez, Ch. (2008). Tercer sector de la comunicación. Teoría y praxis de la televisión alternativa. Una mirada a los casos de España, Estados Unidos y Venezuela. Universidad Autónoma de Barcelona. http://www.tdx.cat/bitstream/ handle/10803/4212/csblde2.pdf

Santoro, D. (2004). Técnicas de Investigación. Métodos desarrollados en diarios y revista de América Latina. México: Fondo de cultura Económica.

Santander, P. (2013). El acceso de los ciudadanos a las noticias de la televisión: la construcción de un tercero discursivo. Revista Literatura y Lingüística $\mathrm{N}^{\circ} 15$, págs.: 183-210. Recuperado de: http://www.scielo. cl/scielo.php?script=sci_arttext\&pid=S0716$58112004001500012 \& \operatorname{lng}=$ es\& $n r m=$ iso

The Commission on Freedom of the Press. (1947). Libertad y Responsabilidad de la Prensa. Universidad de Chicago. Segunda edición.

Verdú, Y. (2009), Sesgo y encuadre en las noticias de televisión. Mecanismos de manipulación periodística a propósito del urbanismo y del agua en los informativos de canal 9. Universidad de Valencia. España. Recuperado de:http://www.tdx.cat/bitstream/ handle/10803/10331/verdu.pdf 